\title{
Efficient Vortex-assisted Extraction of Phorbol Esters from Jatropha Leaves and Correlation Between Leaves and Seeds in Phorbol EstersContent
}

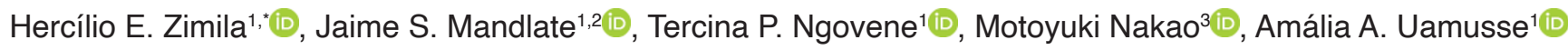 \\ ${ }^{1}$ Department of Chemistry, Eduardo Mondlane University, P.O.Box 257, Main Campus, Maputo, Mozambique \\ ${ }^{2}$ Departamento de Química, Universidade Federal de Santa Maria, 97105-900, Santa Maria, RS, Brazil \\ ${ }^{3}$ Department of Public Health, School of Medicine, Kurume University, 67 Asahimachi, Kurume, Fukuoka 830-0011, Japan
}

Received 22 March 2020, revised 07 May 2021, accepted 16 June 2021

\begin{abstract}
This work aimed to optimise and validate the vortex-assisted extraction with high-performance liquid chromatographyultraviolet detection (HPLC-UV) method to quantify phorbol esters (PEs) in Jatropha leaves. Additionally, to evaluate the correlation between PEs content in leaves and seeds. The results of PEs content were expressed as equivalent of the major Jatropha phorbol ester, 12-deoxy-16-hydroxyphorbol-4'-[12',14'-butadienyl]-6'-[16',18',20'-nonatrienyl]-bicyclo[3.1.0]-hexane(13-O)-2'-[carboxylate]-(16-O)-3'-] $8^{\prime}$-butenoic-10'] ate (DHPB) . One-variable-at-time strategy and $2^{5-1}$ factorial fractional design were employed to determine the effects of solvent type, volume, stirring frequency, extraction time and cycles on yield. The quantification was performed using HPLC-UV. The optimum conditions were three extractions with $2 \%$ tetrahydrofuran:dichloromethane (1:1) in methanol (THF:DCM/MeOH). The sample:solvent ratio was $1 \mathrm{mg}: 25 \mu \mathrm{L}$, and the vortex stirring was $2200 \mathrm{rpm}$ for 5 minutes. The method showed good linearity $\left(R^{2} 0.999\right)$, recoveries $(97$ to $105 \%)$, selectivity and repeatability (RSD 1.93-7.95\%). In addition, good limits of detection and quantification of 2.19 and $6.65 \mathrm{ng} \mu \mathrm{L}^{-1}$, respectively, were noted. DHPB content in leaves and seeds ranged from 0.01 to $0.38 \mathrm{mg} \mathrm{g}^{-1}$ and 0.41 to $1.82 \mathrm{mg} \mathrm{g}^{-1}$, respectively. There is a statistically significant and positive linear relationship between leaves and seeds for DHPB content. These results may have practical application in analysing and predicting the amount of PEs in Jatropha leaves for environmental safety studies and the selection of better varieties for genetic improvement.
\end{abstract}

KEYWORDS

correlation, Jatropha leaves; Jatropha seeds; phorbol esters; vortex-assisted.

\section{Introduction}

Jatropha is a non-edible, drought-resistant, multipurpose oil crop grown in many tropical and subtropical countries. ${ }^{1}$ In developing countries, Jatropha has been promoted especially for biodiesel production to reduce dependence on fossil fuel imports, improving rural livelihoods. . $^{2,3}$

Several Jatropha germplasms have been established worldwide, through which breeding programs have been performed to turn Jatropha into a profitable industrial crop. ${ }^{4,5}$ Intensification of cultivation campaigns of Jatropha has led to a significant increase of contact between humans and the Jatropha plant. This contact imminently leads to occupational health risks due to toxic tigliane diterpenes (referred to as phorbol esters (PEs)) in the whole plant. ${ }^{6}$ These compounds are analogues of diacylglycerol and act by activating the protein kinase $C$, which is involved in various effects, including apoptosis, platelet aggregation, cell differentiation, and tumours. ${ }^{7}$ Studies of Devappa et al. ${ }^{8}$ revealed that PEs exhibited severe alterations towards reconstructed human epithelium and human corneal epithelium, hence are highly toxic in contact with eyes and skin.

On the other hand, Kato and Takechi ${ }^{9}$ reported that tolerable daily intake of PEs for dermal exposure is as low as $0.01 \mu \mathrm{g} \mathrm{kg}^{-1}$. These facts signal the urgent need to assess the human health risks associated with short- and long-term contact with the PEscontaining Jatropha plant. For this purpose, robust and sensitive analytical methods for determining PEs in all Jatropha aerial parts are critical.
Efficient and accurate methods for quantifying PEs in seeds have already been developed, optimised and their fitnessfor-purpose was demonstrated through a comprehensive validation process ${ }^{10}$. Nevertheless, the methods for sample preparation for further determination of PEs in leaves have not yet been well established. To our knowledge, the only approach available is the ultrasound-assisted solid-liquid extraction coupled with chromatographic techniques, reported by Baldini et al. ${ }^{11}$ Technically, this is a noticeably tedious and time-consuming ( $\sim 65 \mathrm{~min})$ strategy whose routine use may raise environmental concerns due to the consumption and disposal of large amounts of organic solvents (about $51 \mathrm{~mL} /$ sample). The amount of ultrasonic energy that reaches the vial with the sample-solvent mixture is strongly affected by the position and geometry of the vial and is often lower than the applied irradiation..$^{12}$ Additionally, an increase in the bath temperature may occur due to the conversion of energy into heat, causing discrepancies in both extraction conditions and yields. ${ }^{12}$ Moreover, this method's optimisation and validation data are not available, which may lead to underestimating the actual content of PEs in leaves.

Vortex agitation represents a low-cost, simple and effective technique to provide the mechanical energy required to enhance the dispersion of extracting solvent within the sample matrix $^{12-14}$. It improves the extraction efficiency through the reduction of the time required to attain partition equilibrium. ${ }^{12,14}$ It has been employed to assist several environmental and food samples preparation techniques. Some of these techniques are dispersive liquid-liquid microextraction, ${ }^{12-14}$ surfactantenhanced-emulsification liquid-liquid microextraction, ${ }^{15}$ 
magnetic dispersive solid-phase microextraction, ${ }^{16,17}$ and solid-liquid extraction. ${ }^{10}$ After the preparation of the samples, it is further analysed for target analytes by chromatographic ${ }^{10,12-15}$ and/or spectroscopic techniques. ${ }^{13,17}$ Therefore, the vortex agitation approach can be a suitable alternative to the previously reported method for extracting PEs from Jatropha leaves.

This study employed the experimental design to optimise the vortex-assisted solid-liquid extraction coupled to HPLC-UV for determining the PEs in Jatropha leaves. Furthermore, the correlation between seeds and leaves in terms of the amount of PE was evaluated.

\section{Experimental}

\subsection{Chemicals}

HPLC grade water $\left(18 \mathrm{M} \Omega \mathrm{cm}^{-1}\right)$ was purified by a Milli-Q system (Milli-Q direct 8.0, Japan). All chemicals were purchased from Fluka Chemika and Skylabs (South Africa) unless otherwise specified. Tetrahydrofuran (THF), ethylacetate (EtAc), ethanol (EtOH) and dichloromethane (DCM) were of analytical grade, whereas methanol $(\mathrm{MeOH})$ and acetonitrile were of HPLC grade. The standard of the major Jatropha phorbol ester, 12-deoxy-16-hydroxyphorbol-4'-[12',14'-butadienyl]$6^{\prime}-\left[16^{\prime}, 18^{\prime}, 20^{\prime}\right.$-nonatrienyl] -bicyclo[3.1.0]hexane-(13-O)-2' [carboxylate]-(16-O)-3'-18'-butenoic-10']ate (DHPB), was prepared in Kurume University, Japan, following the method described by Nakao et al. ${ }^{18}$

\section{Sampling and pre-sample preparation}

Eleven Jatropha accessions from the Jatropha germplasm bank established in Boane district (26을 $\left.20.4^{\prime \prime} \mathrm{S}, 32^{\circ}-21^{\prime} 06.5^{\prime \prime} \mathrm{E}\right)$, Maputo Mozambique, were used. These specimens were authenticated by the Botanical Laboratory of the Eduardo Mondlane University. The samples were air-dried at room temperature, crushed in a mortar (until kernels and leaves became sticky and fine powder, respectively) and stored at $-14^{\circ} \mathrm{C}$ in screw-capped polypropylene tubes.

\section{Extraction of PEs from Jatropha leaves and seeds}

PEs were extracted following the procedure previously described by Zimila et al. ${ }^{10}$ Typically, approximately $40 \mathrm{mg}$ samples were extracted 1-3 times with 1-3 mL aliquots of extraction solvent, under vortex-stirring (ThermoScientific LP Vortex Mixer 0-3200 rpm) at 600-2200 rpm for 1-8 min. The mixture was left for 5.0 min (until clear phase separation) at room temperature, and the supernatant was rota-evaporated to dryness at $40^{\circ} \mathrm{C}$. The residue was recovered with $400 \mu \mathrm{L}$ of methanol, filtrated through a nylon syringe filter $0.22 \mu \mathrm{m}$ and injected into the HPLC-UV system.

Optimisation of the conditions for extraction of PEs from leaves consisted of solvent selection by a one-variable-at-time strategy and the whole factors screening through experimental design. The extraction efficiency of DCM, EtAc, EtOH, $\mathrm{MeOH}$, and THF:DCM/MeOH was evaluated, and the two best solvents were selected for experimental design. The sample:solvent ratio, extraction time, vortex-stirring frequency and extraction cycles were kept at $1 \mathrm{mg}: 50 \mu \mathrm{L}, 3 \mathrm{~min} / \mathrm{cycle}, 3200 \mathrm{rpm}$, and 2 cycles, respectively. The influence of extraction time, stirring frequency, extraction cycles, solvent type and volume was screened by the fractional factorial with resolution $\mathrm{V}\left(2^{5-1} \mathrm{v}\right)$ and two centre points. The factor levels are displayed in Table 1.

To extract PEs from the seed kernels, two extractions with methanol at $1 \mathrm{mg}: 50 \mu \mathrm{L}$ sample:solvent ratio, under vortex stirring at $3200 \mathrm{rpm}$ for $3 \mathrm{~min} /$ cycle were applied..$^{10}$

\section{Chromatographic analysis}

The chromatographic analyses were performed according to Zimila et al. ${ }^{10}$ on a Shimadzu HPLC-UV system consisting of a
Table 1. Factors and levels used in experimental design

\begin{tabular}{|c|c|c|c|}
\hline \multirow{2}{*}{ Factor } & \multicolumn{3}{|c|}{ Factor level } \\
\hline & -1 & 0 & +1 \\
\hline Extraction time (min) (A) & 1.0 & 4.5 & 8.0 \\
\hline Stirring rate (rpm) (B) & 600 & 1400 & 2200 \\
\hline Extraction cycles (C) & 1 & 2 & 3 \\
\hline $\begin{array}{l}\text { Sample:solvent ratio } \\
(\mathrm{mg}: \mu \mathrm{L})(\mathrm{D})\end{array}$ & $1: 25$ & $1: 50$ & $1: 75$ \\
\hline Solvent type (E) & $\mathrm{MeOH}$ & $\begin{array}{c}\mathrm{MeOH} \text { or } \\
\text { THF/DCM/MeOH* }\end{array}$ & THF/DCM/MeOH \\
\hline
\end{tabular}

*In the central point (level 0) of solvent type (categorical variable), either $\mathrm{MeOH}$ or THF/DCM/MeOH was used. Please refer to runs 4 and 10 in Table 2 .

controller SCL-10AVP, degasser CTO-20A, pump LC-20AT and detector SPD-20A. The separation was carried out at $40^{\circ} \mathrm{C}$ on an ODS4 C18 column $(5 \mu \mathrm{m}, 4.6 \mathrm{~mm} \times 150 \mathrm{~mm}$, GLScience Inc., Tokyo), protected by a pre-column with the same composition $(4.0 \mathrm{~mm} \times 10 \mathrm{~mm})$. A mobile phase consisting of a mixture of acetonitrile:water (77:23) was eluated in an isocratic mode at a flow rate of $1.0 \mathrm{~mL} \mathrm{~min}{ }^{-1}$. The wavelength was set at 282 $\mathrm{nm}$ and the run time was $20 \mathrm{~min}$. The chromatographic peaks of PEs were in the range of 10 to $15.5 \mathrm{~min}$, and the methanol solutions of DHPB at 2.5, 5.0, 10.0, 25.0 and $50.0 \mathrm{ng} \mu \mathrm{L}^{-1}$ were used to obtain the calibration curve.

\section{Statistical analysis}

Analysis of Variance (ANOVA) toolbox of Minitab 18.0 statistical software at $95 \%$ confidence level was used for experimental design and data processing. The statistical significance was considered at $\mathrm{p}<0.05$.

\section{Results and Discussion}

Solvent selection for extraction of PEs from Jatropha leaves

The crucial step for efficient extraction and analysis of PEs from leaves is the selection of a suitable solvent. According to Schmidt and Hecker, ${ }^{19}$ the PEs show significant solubility in medium and high polarity organic solvents, such as dimethylsulphoxide (DMSO), dichloromethane (DCM), ethylacetate (EtAc), methanol $(\mathrm{MeOH})$ and ethanol (EtOH). The extraction efficiency of DCM, EtAc, $\mathrm{EtOH}, \mathrm{MeOH}$, and THF:DCM/MeOH was evaluated, and the content of PEs, as an equivalent of DHPB, obtained from each solvent is depicted in Fig 1.

The best DHPB recovery was obtained using $\mathrm{MeOH}$ and THF:DCM/MeOH; hence these solvents were selected for the experimental design. Despite the difference in nature of the matrices, this result is in accordance with previous studies by Devappa et al. ${ }^{20}$ in which $\mathrm{MeOH}$ and THF:DCM/MeOH were

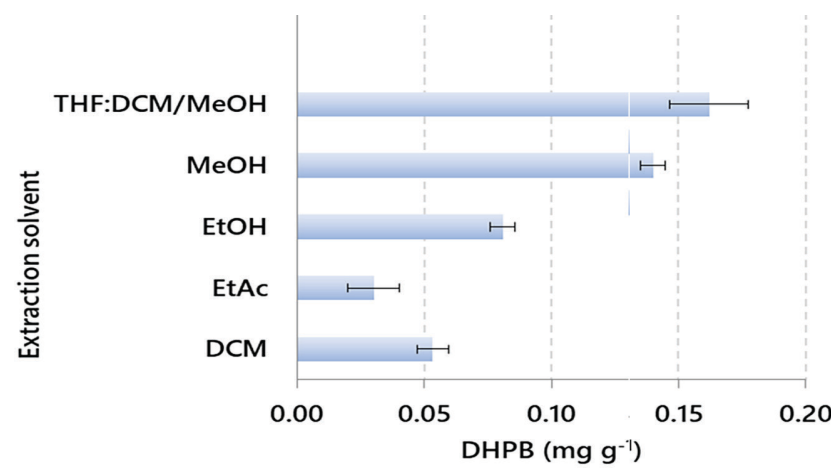

Figure 1. Effect of extraction solvents on the content of DHPB Where: THF:DCM/MeOH is $2 \%$ of tetrahydrofuran:dichloromethane (1:1) in methanol; DCM, EtAc, EtOH and $\mathrm{MeOH}$ are, respectively, dichloromethane, ethyl acetate, ethanol and methanol. 
found to be the best extraction solvents of PEs from Jatropha oil.

Both DCM and EtAc showed low extraction yields and chromatograms with poorly resolved peaks, presumably due to co-extraction of metabolites with retention times close to those of PEs. On the other hand, EtOH exhibited well-resolved chromatograms, but its extraction efficiency was relatively low.

\section{Optimisation of the extraction of DHPB from Jatropha leaves}

Solvent extraction is a series of phenomenological processes strongly affected by various factors, including solvent type, volume, matrix composition, extraction time, sample size, and temperature ${ }^{21}$. Among them, extraction time, stirring frequency, extraction cycles, solvent type and volume were selected for this study, as temperature and pressure are not technically feasible for vortex-assisted extraction. The influence of these factors on the extraction yield was studied by the fractional factorial with resolution $\mathrm{V}\left(2_{\mathrm{V}}^{5-1}\right)$. Two centre points were included to assess the possible nonlinear relationship between variables and extraction yield..$^{22}$ The factor levels were chosen based on preliminary assays in our laboratory. The design matrix and the content of DHPB (expressed as mean of three replicates \pm standard deviation) obtained in each run are shown in Table 2 .

The effects of the studied variables are shown in Fig. 2 as a Pareto chart. In this chart, the length of the bar is proportional to the effect of the variable on the extraction yield. ${ }^{23}$

Factors whose bar lengths extend beyond the critical value of 2.06 are statistically significant at the $95 \%$ confidence level $(p<0.05)$. Thus, extraction time and solvent were the most influential variables, whereas the others had little effect on extraction recovery. On the other hand, five 2-way interactions (extraction time $\times$ stirring rate, extraction time $\times$ sample:solvent ratio, extraction time $\times$ extracting solvent, stirring rate $\times$ sample:solvent ratio, extraction cycles $\times$ sample:solvent ratio and extraction cycles $\times$ extracting solvent) were statistically significant. Graphically, such interactions lacked parallelism (Fig. 3) and were assigned with letters $\mathrm{A}$ to $\mathrm{F}$.

Table 2. $2^{5-1}$ fractional factorial design matrix with the content of DHPB, in mean $\left(\mathrm{mg} \mathrm{g}^{-1}\right) \pm$ standard deviation, obtained in each run.

\begin{tabular}{lcccccc}
\hline Run & A & B & C & D & E & DHPB $\left(\mathrm{mg} \mathrm{g}^{-1}\right)$ \\
\hline 1 & 8 & 2200 & 1 & $1: 25$ & THF:DCM/MeOH & $0.1282 \pm 0.0001$ \\
2 & 1 & 2200 & 3 & $1: 25$ & THF:DCM/MeOH & $0.1021 \pm 0.0044$ \\
3 & 8 & 600 & 1 & $1: 25$ & MeOH & $0.1028 \pm 0.0011$ \\
4 & 4.5 & 1400 & 2 & $1: 50$ & THF:DCM/MeOH & $0.1176 \pm 0.0048$ \\
5 & 1 & 600 & 1 & $1: 75$ & $\mathrm{MeOH}$ & $0.1328 \pm 0.0021$ \\
6 & 8 & 2200 & 1 & $1: 75$ & $\mathrm{MeOH}$ & $0.1037 \pm 0.0104$ \\
7 & 1 & 600 & 3 & $1: 25$ & $\mathrm{MeOH}$ & $0.1144 \pm 0.0037$ \\
8 & 8 & 2200 & 3 & $1: 25$ & $\mathrm{MeOH}$ & $0.1120 \pm 0.0028$ \\
9 & 8 & 2200 & 3 & $1: 75$ & THF:DCM/MeOH & $0.1337 \pm 0.0026$ \\
10 & 4.5 & 1400 & 2 & $1: 50$ & $\mathrm{MeOH}$ & $0.1072 \pm 0.0115$ \\
11 & 1 & 600 & 1 & $1: 25$ & THF:DCM/MeOH & $0.0800 \pm 0.0039$ \\
12 & 8 & 600 & 1 & $1: 75$ & THF:DCM/MeOH & $0.1231 \pm 0.0089$ \\
13 & 8 & 600 & 3 & $1: 75$ & $\mathrm{MeOH}$ & $0.0857 \pm 0.0012$ \\
14 & 1 & 2200 & 1 & $1: 25$ & $\mathrm{MeOH}$ & $0.1175 \pm 0.0031$ \\
15 & 1 & 2200 & 3 & $1: 75$ & $\mathrm{MeOH}$ & $0.1002 \pm 0.0113$ \\
16 & 1 & 600 & 3 & $1: 75$ & THF:DCM/MeOH & $0.1196 \pm 0.0180$ \\
17 & 1 & 2200 & 1 & $1: 75$ & THF:DCM/MeOH & $0.0900 \pm 0.0009$ \\
18 & 8 & 600 & 3 & $1: 25$ & THF:DCM/MeOH & $0.1374 \pm 0.0070$ \\
19 & 8 & 2200 & 1 & $1: 25$ & THF:DCM/MeOH & $0.1282 \pm 0.0001$ \\
20 & 1 & 2200 & 3 & $1: 25$ & THF:DCM/MeOH & $0.1021 \pm 0.0044$ \\
\hline
\end{tabular}

Where: A- Extraction time (min), B - Stirring rate (rpm), C - Extraction cycles, D - Sample:solvent ratio (mg: $\mu \mathrm{L}), \mathrm{E}$ - Solvent type

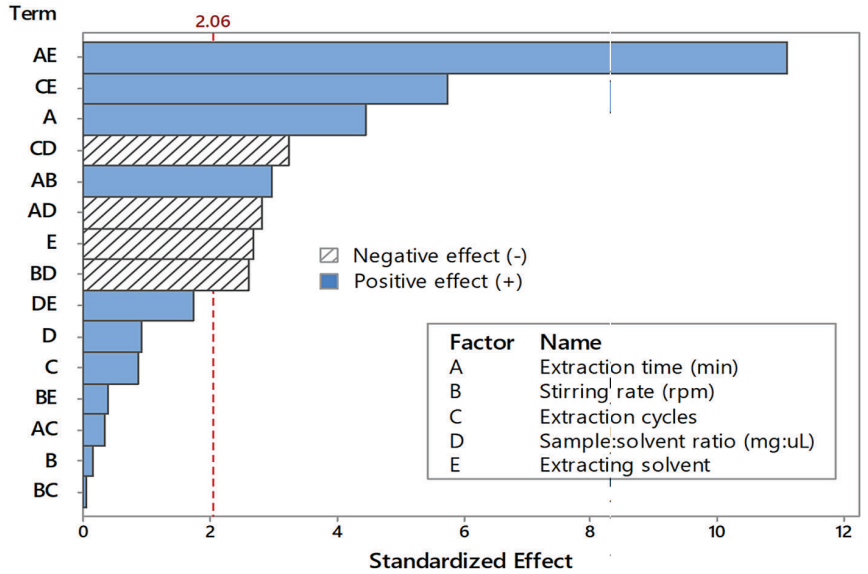

Figure 2. Standardised effects Pareto chart for the $2^{5-1}$ fractional factorial design.

According to Montgomery, ${ }^{24}$ the significance of an interaction between two terms implies that one factor is strongly affected by the level of the other. The interaction of extraction time $\times$ extraction solvent (Fig. 3E) reveals that $\mathrm{MeOH}$ provided higher DHPB recovery than THF:DCM/MeOH when extraction was performed for $1 \mathrm{~min}$. In comparison, THF:DCM/MeOH exhibited higher efficiency than $\mathrm{MeOH}$ when extraction time was extended to 4.5 or $8.0 \mathrm{~min}$. On the other hand, Fig. $3 \mathrm{~A}$ and $3 \mathrm{~B}$ show that when the extraction time was at $8 \mathrm{~min}$, good recoveries were obtained by combining a sample:solvent ratio of 1:25 with vigorous stirring (2200 rpm). In contrast, the extraction was carried out for $1 \mathrm{~min}$, and high recoveries were attained by a low stirring rate $(600 \mathrm{rpm})$ and a sample:solvent ratio of 1:75. These facts imply that the decrease of both polarity and dielectric constant due to the addition of THF:DCM had a notable effect on $\mathrm{MeOH}$ extraction capacity. Perhaps, the modifiers improved their ability to overcome the intermolecular forces binding the DHPB to the matrix, increasing the extraction efficiency. Nevertheless, the time required to attain the highest recovery probably increased because the modifiers decreased the mass transfer rate of PEs to the liquid medium.

Fig. 3F (interaction extraction cycles $\times$ extraction solvent) indicates that good yields were obtained by making several extractions with THF:DCM/MeOH or a single $\mathrm{MeOH}$ extraction. Fig. 3D shows that high yields were achieved with a single extraction at the sample:solvent ratio of 1:75 or three successive extractions with a sample:solvent ratio of $1: 25$. This phenomenon may be related to a drop in $\mathrm{MeOH}$ solubilisation capability due to the addition of modifiers. Regular solvent renewal is of utmost importance to ensure good yields. ${ }^{18}$ However, such results are contrary to Zimila et al. ${ }^{10}$ where adding modifiers slightly reduced the extraction capacity of pure $\mathrm{MeOH}$. These differences are presumably due to differences in the matrix composition.

In short, the conditions for obtaining high extraction yields with $\mathrm{MeOH}$ are single extraction $(\sim 1 \mathrm{~min})$ at the sample:solvent ratio of 1:75 and vortexing at $600 \mathrm{rpm}$. If THF:DCM/MeOH is used, then three successive extractions ( $8 \mathrm{~min} /$ extraction) in the sample:solvent ratio of 1:25 under vortex agitation at $2200 \mathrm{rpm}$ should be applied. These conditions are in accordance with the contours of Fig. 4, in which the red-coloured surfaces represent the maximum yield conditions.

Since extraction time was the unique important continuous variable, further assays were performed at different levels of extraction time $(1,3,5,10,15 \mathrm{~min})$ to determine the minimum time required to achieve good yields. The extraction was performed with THF:DCM/MeOH, at the sample:solvent ratio of 1:25, under stirring at $2200 \mathrm{rpm}$. The results are illustrated 


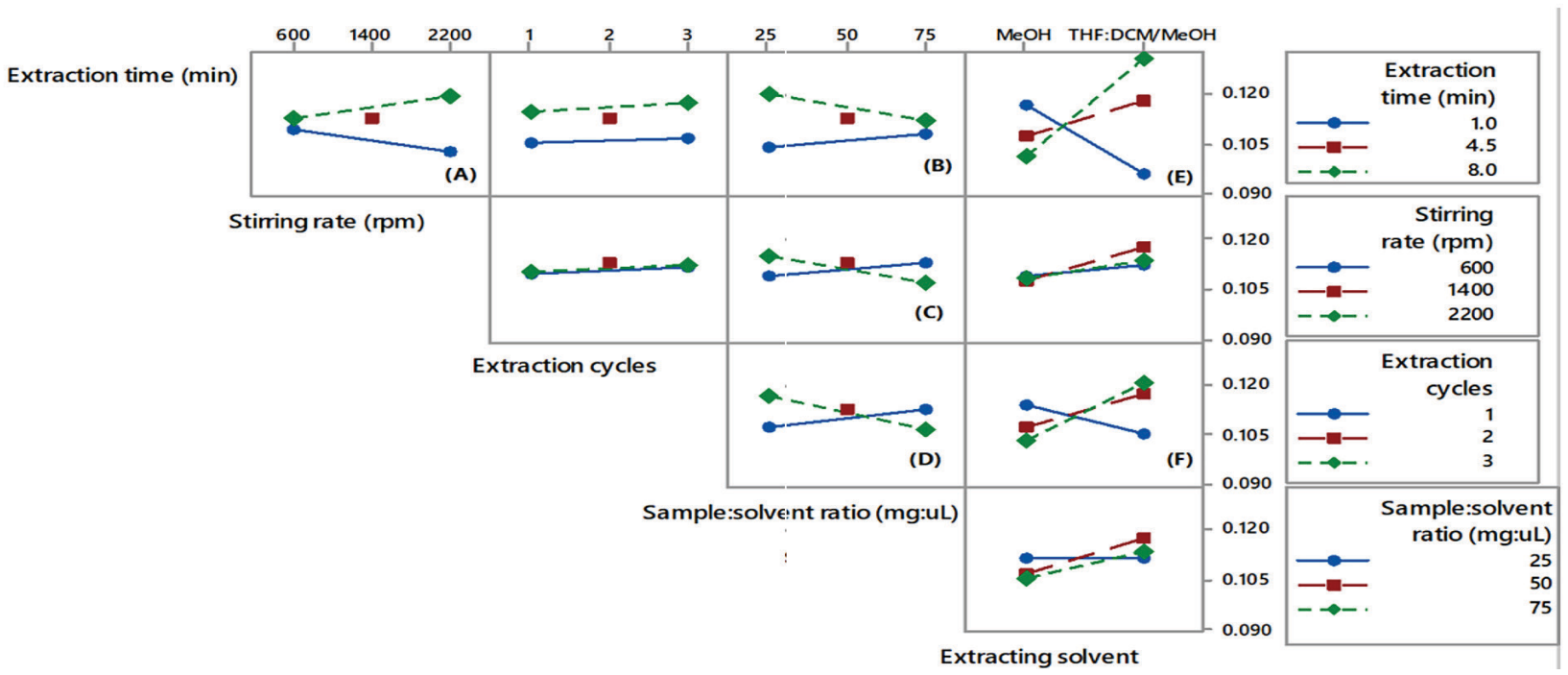

Figure 3. Two-way interaction plots. Plots assigned with letters A to F are statistically significant $(\mathrm{p}<0.05)$. Such plots lack parallelism between the lines, and the effect of one factor depends on the level of the other. The numbers 25, 50, 75 in sample:solvent ratio refer to the ratios of 1:25, 1:50 and 1:50 mg: $\mu \mathrm{L}$ (sample:solvent).
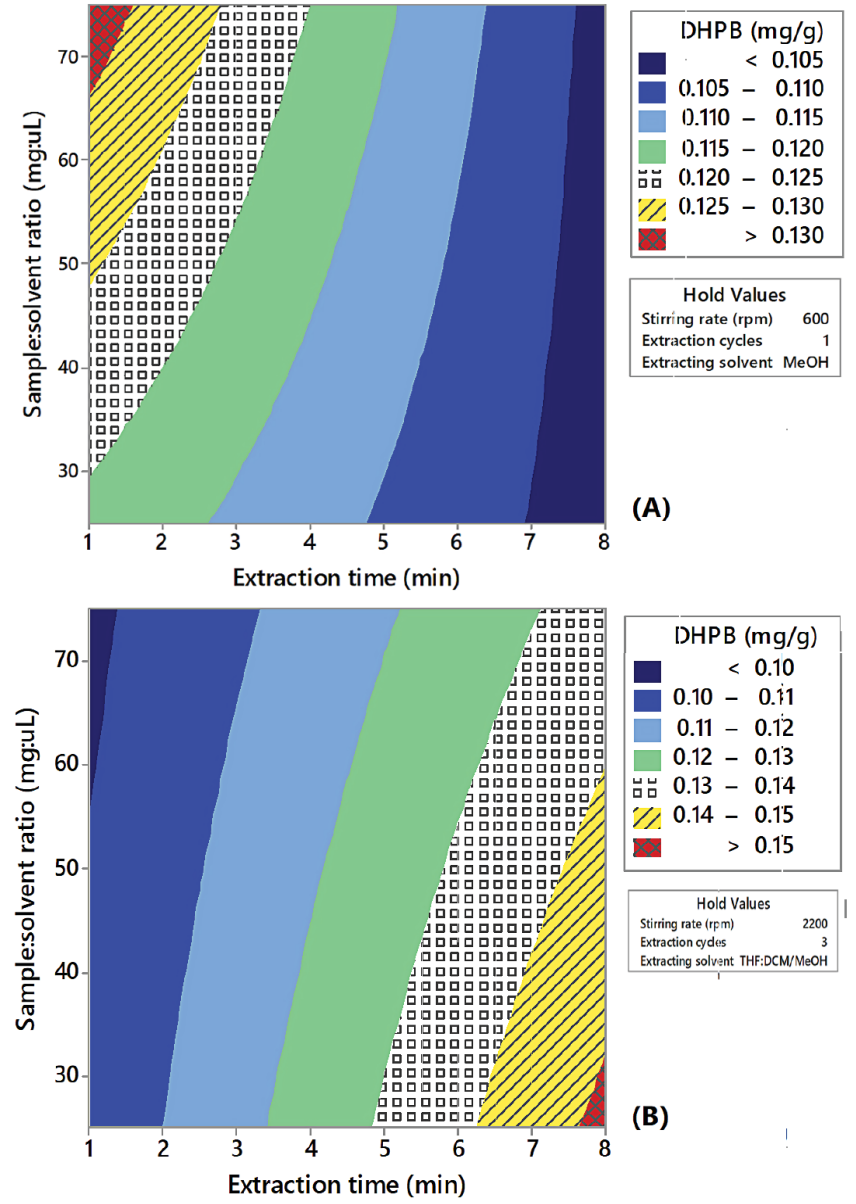

Figure 4. Contour plots for extraction of DHPB from Jatropha leaves with $\mathrm{MeOH}(\mathrm{A})$ and THF:DCM/MeOH (B).

in Fig. 5. ANOVA showed that the yield differences obtained for times 5, 10 and 15 minutes are not statistically significant $(p>0.05)$. Therefore, the optimised method was three extraction cycles (5 min/cycle) with THF:DCM/MeOH, at a sample:solvent ratio of 1:25, under vortex stirring at $2200 \mathrm{rpm}$.

\section{Quality assurance parameters}

The optimised method was validated for linearity,

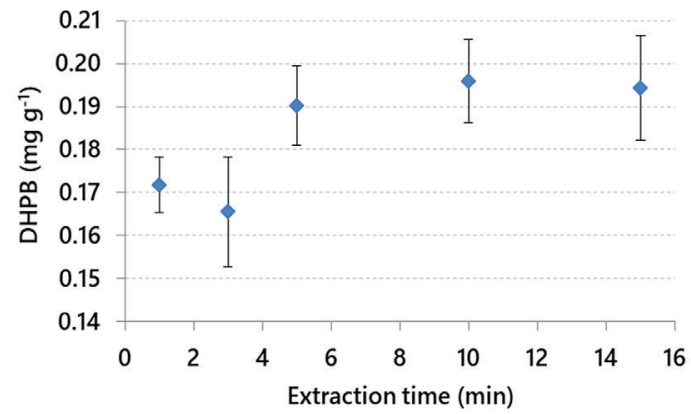

Figure 5. The concentration of DHPB extracted at each extraction time.

selectivity, repeatability, recovery, limits of detection (LOD) and quantification (LOQ), according to Huber ${ }^{25}$ and AOAC International. ${ }^{26}$

Linearity, LOD and LOQ were estimated on the basis of a 5-point calibration curve of DHPB (2.5, 5.0, 10.0, 25.0 and $\left.50.0 \mathrm{ng} \mu \mathrm{L}^{-1}\right)$. The peak areas were found to be linear over the analytical range of 2.5 to $50 \mathrm{ng} \mu \mathrm{L}^{-1}$, with a coefficient of determination $\left(\mathrm{R}^{2}\right)$ of 0.999 . The LOD and LOQ were determined based on the standard deviation of the y-intercept of the regression line $(\sigma)$ and the slope $(S)$, as LOD $=3.3 \sigma / S$ and $\mathrm{LOQ}=10 \sigma / \mathrm{S}$. Their values for LOD and LOQ were 2.19 and $6.65 \mathrm{ng} \mu \mathrm{L}^{-1}$, respectively.

For recovery studies, a PEs-free sample (seeds collected from a non-toxic Jatropha variety) was spiked with a known amount of DHPB and extracted according to the optimised method. The highest DHPB content obtained in the optimisation process $\left(0.15 \mathrm{mg} \mathrm{g}^{-1}\right)$ was assigned the value of $100 \%$. Then, the levels corresponding to $50 \%$ and $150 \%$ were found to be $0.07 \mathrm{mg} \mathrm{g}^{-1}$ and $0.23 \mathrm{mg} \mathrm{g}^{-1}$, respectively. The recovery was expressed as the percentage of DHPB extracted against the amount spiked in the sample. The repeatability was evaluated by calculating the relative standard deviation (RSD) of six replicates at each concentration level. The recoveries were found to be $104.96 \pm 2.11 \%, 102.58 \pm 8.39 \%$ and $96.81 \pm 4.73 \%$ at the concentration levels of $50 \%, 100 \%$ and $150 \%$, respectively. Hence, the method showed both good repeatability (RSD 1.93$7.95 \%$ ) and recoveries.

The method selectivity was assessed by checking the presence of interfering peaks in the retention time range of PEs. The chromatogram of the extracts did not present any additional 
$\mathrm{mV}$

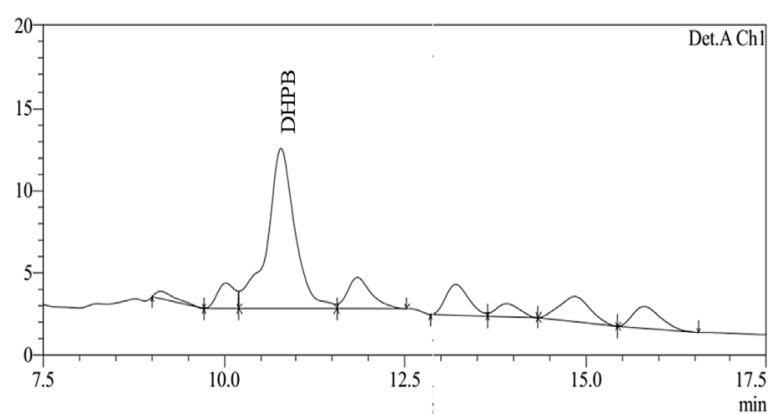

Figure 6. Typical chromatogram of THF:DCM/MeOH extracts of Jatropha leaves.

peaks besides the five reported peaks of PEs in the range of $10-15.5$ min (Fig. 6).

\section{Comparison with previous methods}

The only previously reported method found to determine PEs in Jatropha leaves is the ultrasound-assisted extraction followed by HPLC-UV described by Baldini et al. ${ }^{11}$ The main advantages of the current method (vortex-assisted extraction coupled with HPLC-UV) over the ultrasound-assisted-HPLC-UV include the short extraction time ( $35 \mathrm{~min}$ vs $65 \mathrm{~min}$ ), consumption of a low amount of both extraction solvent ( $3.4 \mathrm{~mL}$ vs $51 \mathrm{~mL}$ ) and sample (40 mg vs $2000 \mathrm{mg}$ ). Both methods showed comparable extraction yields $\left(0.19 \pm 0.01 \mathrm{mg} \mathrm{g}^{-1}\right.$ vs $\left.0.20 \pm 0.01 \mathrm{mg} \mathrm{g}^{-1}\right)$ and selectivity.

\section{Correlation between seeds and leaves in terms of the content of DHPB}

Leaf-seed correlation studies in terms of PEs is a useful tool for predicting the amount of these metabolites in leaves by their content in the seeds, or vice-versa. Eleven Jatropha genotypes, with increasing content of DHPB in seed kernels, were chosen for this study, and the results are presented in Fig. 7.

A strong, positive $(\mathrm{r}=0.927)$ and significant $(\mathrm{p}<0.05)$ linear model, with $\mathrm{R}^{2}$ of 0.806 , was found to be suitable to describe the relationship between leaves and seeds in terms of PEs content. Through this mathematical model, the amount of DHPB in leaves can be deduced from their content in the seeds and viceversa. Nevertheless, an accurate result can only be obtained by an experimental method.

These results imply that if the amount of PEs in the seeds is high, then the PEs amounts in the leaves will also be high. However, similarly to the result reported by Makkar and Becker ${ }^{6}$, the content of PEs in the leaves is lower than in the seeds. The low PEs content in leaves might be attributed to the dilution of PEs during plant growth and/or to the decomposition by environmental conditions since the testa morphologically protects the seed kernel. ${ }^{27}$

\section{Conclusions}

A simple, reliable, cost-effective and fast method for determining PEs in Jatropha leaves has been optimised and validated. Among the five factors considered in this study, only the extraction time and the nature of the solvent had significant effects. The modification of $\mathrm{MeOH}$ with 2\% THF:DCM(1:1) improved its extractive capacity. Jatropha leaves and seeds are strongly correlated with each other in terms of the content of DHPB. The results of the present study evidenced that the optimised method has numerous advantages over the previously reported and has the potential to provide accurate content of PEs in Jatropha leaves for ecotoxicological risk assessments. Furthermore, the content of PEs in Jatropha leaves can be easily deduced by their content in seeds or vice-versa.

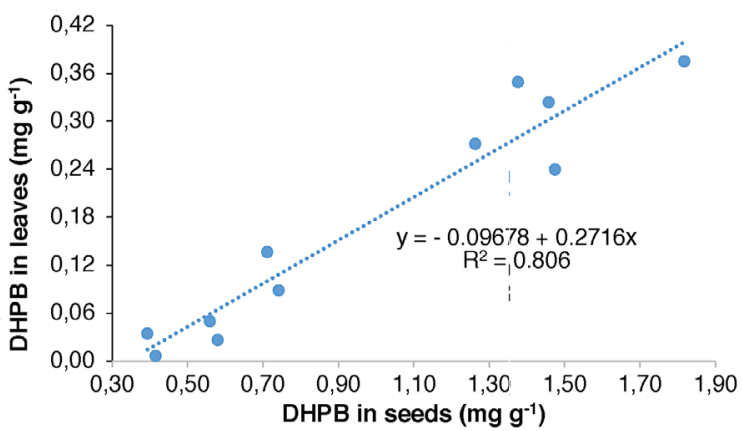

Figure 7. Graphical representation of the relationship between leaves and seeds in DHPB content.

\section{Acknowledgements}

The authors would like to express their sincere gratefulness to Japan International Cooperation Agency (JICA) for financial support of the Project on Sustainable Production of Biodiesel from Jatropha in Mozambique. The authors would also like to thank Amon Tikoko for his help in language and grammar editing.

\section{ORCID iDs}

Motoyuki Nakao: https/orcid.org/0000-0002-1901-554X

Hercilio Zimila: https/orcid.org/0000-0001-5183-6586

Jaime Mandlate: https/orcid.org/0000-0001-6790-5574

Amalia Uamusse: https/orcid.org/0000-0003-4492-4443

Tercina Ngovene: https/orcid.org/0000-002-5850-8750

\section{References}

1 R. Brittaine and N. Lutaladio, Jatropha: A smallholder bioenergy crop The potential for pro-poor development, FAO, Rome, 2010.

2 I. Soto, C. Ellison, M. Kenis, B. Diaz, B. Muys and E. Mathijs, Why do farmers abandon Jatropha cultivation? The case of Chiapas, Mexico, Energy Sustain. Dev., 2018, 42, 77-86.

3 M. Slingerland and M. Schut, Jatropha developments in Mozambique: Analysis of structural conditions influencing niche-regime interactions, Sustainability, 2014, 6, 7541-7563.

4 A.J. King, L.R. Montes, J.G. Clarke, J. Affleck, Y. Li, H. Witsenboer, E. van der Vossen, P. Van Der Linde, Y. Tripathi, E. Tavares, P. Shukla, T. Rajasekaran, E.N. van Loo and I.A. Graham, Linkage mapping in the oilseed crop Jatropha curcas L. reveals a locus controlling the biosynthesis of phorbol esters which cause seed toxicity, Plant Biotechnol. J., 2013, 11, 986-996.

5 C. Yi, C. Reddy, K. Varghese, T. Ngoc, H. Bui, S. Zhang, M. Kallath, B. Kunjachen, S. Ramachandran and Y. Hong, A New Jatropha curcas Variety (JO S2) with Improved Seed Productivity, Sustainability, 2014, 4355-4368.

6 H.P.S. Makkar and K. Becker, Jatropha curcas, a promising crop for the generation of biodiesel and value-added coproducts, Eur. J. Lipid Sci. Technol., 2009, 111, 773-787.

7 G. Goel, H.P.S. Makkar, G. Francis and K. Becker, Phorbol esters: Structure, biological activity, and toxicity in animals, Int. J. Toxicol. 2007, 26, 279-288.

8 R.K. Devappa, J.S. Roach, H.P.S. Makkar and K. Becker, Occular and dermal toxicity of Jatropha curcas phorbol esters, Ecotoxicol. Environ. Saf., 2013, 94, 172-178.

9 J. Kato and S. Takechi, Utilisation of Jatropha curcas: Toxicity of phorbol esters and risk management, J. Japanese Soc. Risk Res., 2015, 24, 221-230.

10 H.E. Zimila, J.S. Mandlate, R.S. Chivodze, H.F. Muiambo, V. Skripets and A.A. Uamusse, An optimised solid-liquid method for rapid extraction of phorbol esters from Mozambican Jatropha seeds, Ind. Crops Prod., 2018, 124, 941-946.

11 M. Baldini, C. Ferfuia, R. Bortolomeazzi, G. Verardo, J. Pascali, E. Piasentier and L. Franceschi, Determination of phorbol esters in seeds and leaves of Jatropha curcas and in animal tissue by high- 
performance liquid chromatography tandem mass spectrometry, Ind. Crops Prod., 2014, 59, 268-276.

12 E. Psillakis, Vortex-assisted liquid-liquid microextraction revisited, Trends Anal. Chem., 2018, 113, 332-339.

13 C.B. Ojeda, F.S. Rojas, Vortex-assisted liquid-liquid microextraction (VALLME): The latest applications, Chromatographia, 2018, 81, 89103.

14 R. Zhang, Z.-C. Tan, K.-C. Huang, Y. Wen, X.-Y. Li, J.-L. Zhao, C.L. Liu, A vortex-assisted dispersive liquid-liquid microextraction followed by UPLC-MS/MS for simultaneous determination of pesticides and aflatoxins in herbal tea, Molecules, 2019, 24, 1029.

15 Z. Yang, Y. Lu, Y. Liu, T. Wu, Z. Zhou, D. Liu, Vortex-assisted surfactant-enhanced-emulsification liquid-liquid microextraction, $J$. Chromatogr. A., 2011, 1218, 7071-7077.

16 X. Cheng, H. Yan, X. Wang, N. Sun, X. Qiao, Vortex-assisted magnetic dispersive solid-phase microextraction for rapid screening and recognition of dicofol residues in tea products, Food Chem., 2014, 162 104-109.

17 E. Yılmaz, M. Soylak, Preparation and characterisation of magnetic carboxylated nanodiamonds for vortex-assisted magnetic solidphase extraction of ziram in food and water samples, Talanta, 2016, $158,152-158$.

18 M. Nakao, G. Hasegawa, T. Yasuhara, Y. Ishihara, Degradation of Jatropha curcas phorbol esters derived from Jatropha oil cake and their tumor-promoting activity, Ecotoxicol. Environ. Saf., 2015, 114 357-364.
19 R. Schmidt, E. Hecker, Autoxidation of phorbol esters under normal storage conditions, Cancer Res., 1975, 35, 1375-1377.

20 R.K. Devappa, H.P.S. Makkar, K. Becker, Optimisation of conditions for the extraction of phorbol esters from Jatropha oil, Biomass and Bioenergy, 2010, 34, 1125-1133.

21 M. D. L. De Castro and J. L. L. García, Acceleration and Automation of Solid Sample Treatment, vol. 24, Elsevier Science B.V., 2002, pp. 233279.

22 T. Lundstedt, E. Seifert, L. Abramo, B. Thelin, Å. Nyström, J. Pettersen, R. Bergman, Experimental design and optimization, Chemom. Intell. Lab. Syst., 1998, 42, 3-40.

23 S. Khodadoust, M. Hadjmohammadi, Determination of $\mathrm{N}$-methylcarbamate insecticides in water samples using dispersive liquid-liquid microextraction and HPLC with the aid of experimental design and desirability function, Anal. Chim. Acta. 2011, 699, 113-119.

24 D.C. Montgomery, Design and analysis of experiments, 8th ed., John Wiley \& Sons, Inc., USA, 2013.

25 L. Huber, Validation of analytical methods, Agilent Technologies, New York, 2010.

26 AOAC International, Guidelines for single laboratory validation of chemical methods for dietary supplements and botanicals, 2002, 38. www. aoac.org.

$27 \mathrm{~S}$. Cleemput, Breeding for a reduced glucosinolate content in the green mass of rapeseed to improve its suitability for biogas production, PhD thesis, University of Göttingen, Germany, 2011. 